Mathematical Research Letters 6, 237-249 (1999)

\title{
MAXIMAL FUNCTIONS AND HILBERT TRANSFORMS ALONG VARIABLE FLAT CURVES
}

\author{
Anthony Carbery and Sonsoles PÉrez
}

\begin{abstract}
In this work we establish $L^{p}$ boundedness for maximal functions and Hilbert transforms along variable curves in the plane, via $L^{2}$ estimates for certain singular integral operators with oscillatory terms.
\end{abstract}

\section{$\S 1$. Introduction}

In this paper, we study the $L^{p}\left(\mathbb{R}^{2}\right)$ boundedness for the maximal function $\mathcal{M}$ and the Hilbert transform $\mathcal{H}$ along variable curves. In our discussions, these are defined a priori on functions in $C_{0}^{\infty}\left(\mathbb{R}^{2}\right)$ by

$$
\mathcal{M} f(x)=\sup _{0<h<\infty} \frac{1}{h}\left|\int_{0}^{h} f\left(x_{1}-t, x_{2}-S\left(x_{1}, x_{1}-t\right)\right) d t\right|
$$

and

$$
\mathcal{H} f(x)=p . v \cdot \int_{-\infty}^{\infty} f\left(x_{1}-t, x_{2}-S\left(x_{1}, x_{1}-t\right)\right) \frac{d t}{t},
$$

where $S(x, y)$ is a suitable real-valued function vanishing on the diagonal.

We shall also consider the singular integral operators $T_{\lambda}$ (acting on functions on the real line) which are of the form

$$
T_{\lambda} f(x)=\lim _{\epsilon \rightarrow 0} \int_{|x-y| \geq \epsilon} e^{i \lambda S(x, y)}(x-y)^{-1} f(y) d y .
$$

Local versions of the operators $T_{\lambda}$ have been studied by Phong and Stein [PS], and by Pan $[\mathrm{P}]$ who proved the $L^{p}$ boundedness of $T_{\lambda}$ with bounds independent of $\lambda$ when the mixed derivative $S_{x y}^{\prime \prime}$ does not vanish to infinite order at any diagonal point $\left(x_{0}, x_{0}\right)$. In [S] Seeger showed that for a certain class of phases $S$ without this finite type property, the associated operators $T_{\lambda}$ are also uniformly bounded on $L^{2}$. Here we extend the Seeger-type result, for a different, closely related (but not always directly comparable) class of phases, to all other $p$, $1<p<\infty$.

Received March 1, 1999.

The second author was partially supported by grant PB97/0030 and both authors were supported by European Commission TMR "Harmonic Analysis" 
Theorem 1. Let $S$ be an antisymmetric function in $C^{3}\left(\mathbb{R}^{2}\right)$. Let $g: \mathbb{R}_{+} \rightarrow \mathbb{R}_{+}$ be a non-decreasing function satisfying that there exists a constant $B \geq 1$ such that

$$
g(B t) \geq 2 g(t)
$$

and suppose that for some $E \geq 1$ and $A \geq 1$,

$$
\begin{aligned}
& A^{-1} g(|x-y|) \leq\left|S_{1}^{\prime}(x, y)\right| \leq A g(E|x-y|), \\
& A^{-1} g(|x-y|) \leq\left|S_{2}^{\prime}(x, y)\right| \leq A g(E|x-y|) .
\end{aligned}
$$

Suppose furthermore that $S_{112}^{\prime \prime \prime}$ is single-signed on $\mathbb{R}^{2}$. Then $\mathcal{M}$ is bounded on $L^{p}$ for all $1<p \leq \infty$, and $\mathcal{H}$ is bounded on $L^{p}$ for all $1<p<\infty$.

A local version of the theorem where the hypotheses are assumed on $S$ in a neighbourhood of the diagonal and $\mathcal{M}$ and $\mathcal{H}$ are suitably modified also holds. Included in this setting are examples such as $S(x, y)=e^{-(x-y)^{-2}} g(x, y)$, $g(x, x) \neq 0$, or $S(x, y)=e^{-(x-y)^{-2} h(x, y)}, h(x, x)>0$, (defined thus for $y>x$ and extended to be antisymmetric) where $g$ and $h$ are smooth. Thus the theorem covers certain "flat" curves which is a point of principal interest.

It is well-known that estimates for $\mathcal{H}$ yield uniform estimates for $T_{\lambda}$. Indeed, if $\mathcal{F}_{2}$ denotes the Fourier transform in the second variable then $\mathcal{F}_{2} \mathcal{H} f\left(x_{1}, \lambda\right)=$ $T_{\lambda}\left(\mathcal{F}_{2} f(\cdot, \lambda)\right)\left(x_{1}\right)$. By applying Plancherel's theorem we see that

$$
\sup _{\lambda \in \mathbb{R}}\left\|T_{\lambda}\right\|_{L^{2}(\mathbb{R}) \rightarrow L^{2}(\mathbb{R})}=\|\mathcal{H}\|_{L^{2}\left(\mathbb{R}^{2}\right) \rightarrow L^{2}\left(\mathbb{R}^{2}\right)} .
$$

Moreover, a variant of de Leeuw's theorem implies that if $\mathcal{H}$ is bounded on $L^{p}\left(\mathbb{R}^{2}\right)$ then

$$
\sup _{\lambda \in \mathbb{R}}\left\|T_{\lambda}\right\|_{L^{p}(\mathbb{R}) \rightarrow L^{p}(\mathbb{R})} \leq\|\mathcal{H}\|_{L^{p}\left(\mathbb{R}^{2}\right) \rightarrow L^{p}\left(\mathbb{R}^{2}\right)} .
$$

Thus, an immediate consequence of Theorem 1 is the following corollary:

Corollary 2. If $S$ satisfies the same hypotheses as in Theorem 1, then for any $p, 1<p<\infty$, we have

$$
\sup _{\lambda \in \mathbb{R}}\left\|T_{\lambda}\right\|_{L^{p}(\mathbb{R}) \rightarrow L^{p}(\mathbb{R})} \leq C .
$$

In the work of Seeger [S], $S$, in addition to (1), is assumed to satisfy a condition on its second order derivatives (related to the so-called $k$-quasimonotonicity condition.) We, in contrast, demand a condition on the third-order derivative $S_{112}^{\prime \prime \prime}$.

In the translation-invariant setting, when $S(x, y)=\gamma(x-y)$, our condition reduces to $\gamma^{\prime \prime \prime} \geq 0$ (see $[\mathrm{DR}]$ ) which implies the infinitessimal doubling (see [CCVWW]) and hence doubling of $\gamma^{\prime}$, (see [NVWW1], [NVWW2] and [CCCDRVWW]). On the other hand, the single-signedness of $S_{112}^{\prime \prime \prime}$ is the minimal 
hypothesis for our proof - based upon smoothing estimates for relatives of $T_{\lambda}$ where the Hilbert singularity is replaced by a smooth cut-off function - to work according to what is currently known about smoothing for oscillatory integral operators. (See for example [PS1] and [CCW]). A general result for more general versions of $\mathcal{H}$ in which the variable curve has rotational curvature not vanishing to infinite order has recently been established in [CNSW]. In our setting, that condition reduces to $S_{x y}^{\prime \prime}$ not vanishing to infinite order on the diagonal. The first results for the non-translation invariant flat case (i.e. in which the rotational curvature may vanish to infinite order on the diagonal) were obtained in [CWW2].

The idea of the proof is to consider pieces of the operators like the following

$$
S_{j} f(x)=\frac{1}{2^{j+1}} \int_{2^{j}<|t| \leq 2^{j+1}} f\left(x_{1}-t, x_{2}-S\left(x_{1}, x_{1}-t\right)\right) d t
$$

and

$$
T_{j} f(x)=\int_{2^{j}<|t| \leq 2^{j+1}} f\left(x_{1}-t, x_{2}-S\left(x_{1}, x_{1}-t\right)\right) \frac{d t}{t} .
$$

Thus $\mathcal{H} f(x)=\sum_{j} T_{j} f(x)$ and $\mathcal{M}$ is bounded for a non-negative function $f$ by $\mathcal{M} f(x) \leq C \sup _{j}\left|S_{j} f(x)\right|$. And then we can apply the following generalization of the Cotlar-Stein lemma, see [C].

Proposition 3. (Almost-Orthogonality Principle) Assume that $\left\{Q_{j}\right\}$ satisfies $\sum_{j \in \mathbb{Z}} Q_{j}=I$. Assume that

$$
\left\|Q_{j}^{*} Q_{k}\right\|_{2-2}+\left\|Q_{j} Q_{k}^{*}\right\|_{2-2} \leq C 2^{-\epsilon|j-k|}
$$

and that

$$
\left\|\sum_{j} \pm Q_{j}\right\|_{p_{0}-p_{0}}+\left\|\sum_{j} \pm Q_{j}^{*}\right\|_{p_{0}-p_{0}} \leq C
$$

for some $p_{0} \in(1,2)$. Suppose that $Q_{j}=P_{j}-P_{j+1}$ where $P_{j} \geq 0$ and $\left\|\sup _{j}\left|P_{j} f\right|\right\|_{r} \leq C_{r}\|f\|_{r}$ for $p_{0} \leq r \leq 2$. Assume also that

$$
\left\|\left(\sum_{k}\left|Q_{k} g_{k}\right|^{2}\right)^{1 / 2}\right\|_{p_{0}^{\prime}} \leq C\left\|\left(\sum_{k}\left|g_{k}\right|^{2}\right)^{1 / 2}\right\|_{p_{0}^{\prime}} .
$$

Suppose that $\left\{T_{j}\right\},\left\{S_{j}\right\}$ satisfy

$$
\left|T_{j} f\right| \leq S_{j}|f|
$$

where $S_{j} \geq 0$. Assume that $\left\|S_{j}\right\|_{r-r} \leq C$ for $p_{0} \leq r \leq 2$. Moreover, assume that

$$
\left\|\left(S_{j}-P_{j}\right) Q_{j+k}^{*}\right\|_{2-2}+\left\|\left(S_{j}-P_{j}\right)^{*} Q_{j+k}\right\|_{2-2} \leq C 2^{-\epsilon|k|},
$$


and

$$
\left\|T_{j} Q_{j+k}^{*}\right\|_{2-2}+\left\|T_{j}^{*} Q_{j+k}\right\|_{2-2} \leq C 2^{-\epsilon|k|} .
$$

Then $f \rightarrow \sup _{j}\left|S_{j} f(x)\right|$ and $\sum_{j} T_{j}$ are bounded on $L^{p}, p_{0}<p \leq 2$.

In order to define the appropriate Littlewood-Paley decomposition $I=\sum_{j} Q_{j}$ we define the dilations,

$$
A(t)=\left(\begin{array}{cc}
t & 0 \\
0 & G(t)
\end{array}\right) \quad \text { with } \quad G(t)=\int_{0}^{t} g(s) d s .
$$

Similar dilations were first explicitly used in the flat translation invariant case in [CCVWW]. The collection $\{A(t)\}$ satisfies the Rivière condition

$$
\left\|A(s)^{-1} A(t)\right\| \leq C\left(\frac{t}{s}\right)^{\epsilon} \quad \text { for } \quad s \geq t, \quad \text { for some } \quad \epsilon>0 .
$$

In fact in this case it is true with $\epsilon=1$ and it is enough to show that $\frac{G(t)}{G(s)} \leq C \frac{t}{s}$ for $s \geq t$. Observe that if $s \geq t$ there exists a natural number $k$ such that $B^{k} t \leq s \leq B^{k+1} t$ and we have

$$
\begin{aligned}
\frac{1}{t} G(t) & =\frac{1}{t} \int_{0}^{t} g(u) d u \leq \frac{1}{2 t} \int_{0}^{t} g(B u) d u \leq \cdots \leq \frac{1}{2^{k} t} \int_{0}^{t} g\left(B^{k} u\right) d u \\
& =\frac{1}{B^{k} 2^{k} t} \int_{0}^{B^{k} t} g(u) d u \leq \frac{1}{B^{k} 2^{k} t} G(s)=\frac{s}{t} \frac{1}{B^{k} 2^{k}} \frac{G(s)}{s} \leq B \frac{G(s)}{s} .
\end{aligned}
$$

Let $\phi$ be a nonnegative $C_{0}^{\infty}\left(\mathbb{R}^{2}\right)$ function such that $\int \phi=1$. We set the initial averaging operator

$$
P_{0} f(x)=\int \phi(x-y) f(y) d y
$$

then an approximation of the identity (with $P_{j} \rightarrow I$ as $j \rightarrow-\infty$ and $P_{j} \rightarrow 0$ as $j \rightarrow \infty)$ is given by

$$
P_{j} f(x)=\int\left(\operatorname{det} A_{j}\right)^{-1} \phi\left(A_{j}^{-1}(x-y)\right) f(y) d y \quad \text { with } \quad A_{j}=A\left(2^{j}\right) .
$$

The natural Littlewood-Paley difference operators $Q_{j}$ are then $Q_{j}=P_{j}-P_{j+1}$.

According to [CVWW] and [CWW1], the conditions on the operators $P_{j}$ and $Q_{j}$ in the almost orthogonality lemma are satisfied for any $p_{0}, 1<p_{0}<\infty$; just the Rivière condition is required. Therefore, subject to having verified (2) and (3), Proposition 3 shows that $\mathcal{M}$ and $\mathcal{H}$ are bounded on $1<p \leq 2$. But the maximal function is trivially bounded on $L^{\infty}$, thus it maps continuously $L^{p}$ into $L^{p}$ for $1<p \leq \infty$. And for $\mathcal{H}$ we notice that the original problem itself is selfadjoint, so the boundedness of $\mathcal{H}$ for $1<p \leq 2$ implies its boundedness for $2 \leq p<\infty$.

It hence remains to prove (2) and (3). 


\section{The curves and their normalization}

Let $S(x, y)$ be an antisymmetric function. Then it is easy to see that $S_{1}^{\prime}(z, x)=-S_{2}^{\prime}(x, z), S_{11}^{\prime \prime}(z, x)=-S_{22}^{\prime \prime}(x, z), S_{12}^{\prime \prime}(z, x)=-S_{12}^{\prime \prime}(x, z)$ (consequently $\left.S_{12}^{\prime \prime}(x, x)=0\right)$, and $S_{112}^{\prime \prime \prime}(z, x)=-S_{122}^{\prime \prime \prime}(x, z)$. Since we also assume that $S_{112}^{\prime \prime \prime}$ does not change sign then $S_{122}^{\prime \prime \prime}$ does not change its sign either and its sign is opposite to that of $S_{112}^{\prime \prime \prime}$. On the other hand, by applying the mean value theorem we get

$$
\operatorname{sgn} S_{12}^{\prime \prime}(z, x)=\operatorname{sgn} S_{112}^{\prime \prime \prime} \operatorname{sgn}(z-x) .
$$

Finally, by using $S_{1}^{\prime}(x, x)=S_{2}^{\prime}(x, x)=0$ one may see that

$$
\operatorname{sgn} S_{1}^{\prime}=-\operatorname{sgn} S_{112}^{\prime \prime \prime} \quad \text { and } \quad \operatorname{sgn} S_{2}^{\prime}=\operatorname{sgn} S_{112}^{\prime \prime \prime},
$$

since $S_{1}^{\prime}(z, x)=S_{12}^{\prime \prime}(z, \nu)(x-z)$ for some $\nu \in \overline{z x}$ (the line segment joining $z$ to $x)$ and so $\left.\operatorname{sgn} S_{1}^{\prime}(z, x)=\operatorname{sgn} S_{112}^{\prime \prime \prime} \operatorname{sgn}(z-\nu) \operatorname{sgn}(x-z)\right)$.

Lemma 4. If $S_{112}^{\prime \prime \prime}$ is single-signed and $S$ is antisymmetric then for any $x$ and

$y$

$$
\left|S_{1}^{\prime}(x, y)\right| \leq|x-y|\left|S_{12}^{\prime \prime}(x, y)\right| \quad \text { and } \quad\left|S_{2}^{\prime}(x, y)\right| \leq|x-y|\left|S_{12}^{\prime \prime}(x, y)\right| .
$$

Proof. We use the mean value theorem:

$$
\left|S_{1}^{\prime}(x, y)\right|=\left|S_{1}^{\prime}(x, y)-S_{1}^{\prime}(x, x)\right|=\left|S_{12}^{\prime \prime}(x, u)\right||x-y| \quad \text { for some } \quad u \in \overline{x y}
$$

If $x<y$ then $x<u<y$ and $\left|S_{12}^{\prime \prime}(x, u)\right|=-\operatorname{sgn} S_{112}^{\prime \prime \prime} S_{12}^{\prime \prime}(x, u)$. Since $\operatorname{sgn} S_{112}^{\prime \prime \prime}=$ $-\operatorname{sgn} S_{122}^{\prime \prime \prime}$ this function is increasing in $u$ and so $\left|S_{12}^{\prime \prime}(x, u)\right| \leq\left|S_{12}^{\prime \prime}(x, y)\right|$. When $x>y,\left|S_{12}^{\prime \prime}(x, u)\right|=\operatorname{sgn} S_{112}^{\prime \prime \prime} S_{12}^{\prime \prime}(x, u)$ is decreasing in $u$ and thus also $\left|S_{12}^{\prime \prime}(x, u)\right| \leq\left|S_{12}^{\prime \prime}(x, y)\right|$.

To prove the estimate for $\left|S_{2}^{\prime}(x, y)\right|$ we can repeat the proof, or realize that $\left|S_{2}^{\prime}(x, y)\right|=\left|S_{1}^{\prime}(y, x)\right| \leq|y-x|\left|S_{12}^{\prime \prime}(y, x)\right|=|y-x|\left|S_{12}^{\prime \prime}(x, y)\right|$, since $S_{12}^{\prime \prime}$ is also antisymmetric.

In our development we shall need to work with normalized versions of $S(.,$.$) ,$ that is, for fixed $j$

$$
\tilde{S}(x, y)=\frac{S\left(2^{j} x, 2^{j} y\right)}{G\left(2^{j}\right)}
$$

It is easy to check several facts concerning them that we shall need later on. First,

$$
\text { for } i=1,2 \quad\left|\tilde{S}_{i}^{\prime}(x, y)\right| \geq A^{-1} \quad \text { whenever } \quad|x-y| \geq 1 .
$$

To see this observe that

$$
\left|\tilde{S}_{i}^{\prime}(x, y)\right|=\frac{2^{j}\left|S_{i}^{\prime}\left(2^{j} x, 2^{j} y\right)\right|}{G\left(2^{j}\right)} \geq \frac{A^{-1} 2^{j} g\left(\left|2^{j} x-2^{j} y\right|\right)}{G\left(2^{j}\right)} \geq \frac{A^{-1} 2^{j} g\left(2^{j}\right)}{G\left(2^{j}\right)} \geq A^{-1},
$$


where the last inequality is true because $g$ is a non-decreasing function.

By Lemma 4 whenever $|x-y| \leq C_{0}$ then

$$
\left|\tilde{S}_{1}^{\prime}(x, y)\right| \leq C_{0}\left|\tilde{S}_{12}^{\prime \prime}(x, y)\right| \quad \text { and } \quad\left|\tilde{S}_{2}^{\prime}(x, y)\right| \leq C_{0}\left|\tilde{S}_{12}^{\prime \prime}(x, y)\right|
$$

since

$$
\left|\tilde{S}_{1}^{\prime}(x, y)\right|=2^{j} \frac{\left|S_{1}^{\prime}\left(2^{j} x, 2^{j} y\right)\right|}{G\left(2^{j}\right)} \leq 2^{j}\left|2^{j} x-2^{j} y\right| \frac{\left|S_{12}^{\prime \prime}\left(2^{j} x, 2^{j} y\right)\right|}{G\left(2^{j}\right)}=\left|x-y \| \tilde{S}_{12}^{\prime \prime}(x, y)\right| .
$$

With this observation we can prove the following lemma:

Lemma 5. If $S$ is antisymmetric and $S_{112}^{\prime \prime \prime}$ is single-signed then, for any $x, y$ and $z$ such that either $-C_{1} \leq z-x, z-y \leq 0$ with $x \leq y$, or $0 \leq z-x, z-y \leq C_{1}$ for $x \geq y$, there exists a constant $C$ such that

$$
\frac{\left|\tilde{S}_{1}^{\prime}(z, y)\right|+\left|\tilde{S}_{1}^{\prime}(z, x)\right|+\left|\tilde{S}_{2}^{\prime}(z, x)\right|}{\left|\tilde{S}_{1}^{\prime}(z, x)-\tilde{S}_{1}^{\prime}(z, y)\right|} \leq \frac{C}{|x-y|} .
$$

Proof. We consider the case $-C_{1} \leq z-x, z-y \leq 0$ which implies $|x-y| \leq 2 C_{1}$ with $x \leq y$ (the proof for the case $0 \leq z-x, z-y \leq C_{1}$ for $x \geq y$ is a repetition of the following arguments). We have that

$$
\begin{aligned}
\mid \tilde{S}_{1}^{\prime}(z, x) & -\tilde{S}_{1}^{\prime}(z, y) \mid=\int_{x}^{y}-\operatorname{sgn} \tilde{S}_{112}^{\prime \prime \prime} \cdot \tilde{S}_{12}^{\prime \prime}(z, u) d u \\
& \geq-\operatorname{sgn} \tilde{S}_{112}^{\prime \prime \prime} \cdot \tilde{S}_{12}^{\prime \prime}(z, x)(y-x)=\left|\tilde{S}_{12}^{\prime \prime}(z, x)\right||y-x|,
\end{aligned}
$$

where we have used that the function inside the integral is increasing in $u$. By (7), as $|z-x| \leq C_{1}$ then

$$
\left|\tilde{S}_{1}^{\prime}(z, x)-\tilde{S}_{1}^{\prime}(z, y)\right| \geq c\left|\tilde{S}_{1}^{\prime}(z, x)\right||y-x|
$$

and

$$
\left|\tilde{S}_{1}^{\prime}(z, x)-\tilde{S}_{1}^{\prime}(z, y)\right| \geq c\left|\tilde{S}_{2}^{\prime}(z, x)\right||y-x| .
$$

Then we just need to prove the lemma for $\left|\tilde{S}_{1}^{\prime}(z, y)\right|$. If $\left|\tilde{S}_{1}^{\prime}(z, x)\right| \geq \frac{\left|\tilde{S}_{1}^{\prime}(z, y)\right|}{2}$ then with the previous estimate we get also $\left|\tilde{S}_{1}^{\prime}(z, x)-\tilde{S}_{1}^{\prime}(z, y)\right| \geq c\left|\tilde{S}_{1}^{\prime}(z, y)\right||y-x|$. But otherwise $\left|\tilde{S}_{1}^{\prime}(z, x)-\tilde{S}_{1}^{\prime}(z, y)\right| \geq \frac{1}{2}\left|\tilde{S}_{1}^{\prime}(z, y)\right| \geq c\left|\tilde{S}_{1}^{\prime}(z, y)\right||y-x|$, since $|y-x| \leq$ $2 C_{1}$.

\section{The heart of the proof}

If $T$ is an integral operator on $\mathbb{R}^{n}$ with distribution kernel $K(x, y)$, and $A \in$ $G L(n, \mathbb{R})$, we let $A_{*} T$ be the operator whose kernel is $(\operatorname{det} A)^{-1} K\left(A^{-1} x, A^{-1} y\right)$. Thus $\left\|A_{*} T\right\|_{p-p}=\|T\|_{p-p}$ for all $1 \leq p \leq \infty$. In the case that $T$ is the Hilbert 
transform along a curve $\Gamma(x, t)$, then $A_{*} T$ becomes the Hilbert transform along the curve $A_{*} \Gamma$, where $\left(A_{*} \Gamma\right)(x, t)=A\left[\Gamma\left(A^{-1} x, t\right)\right]$.

We just need to prove estimates (2) and (3). By the essential self-adjointness of the problem, it suffices to prove either the first or the second inequalities in (2) and (3). For $k>0$, they are a direct consequence of the smoothness of $\left\{P_{j}\right\}$, the support properties of $\left\{T_{j}, S_{j}, P_{j}\right\}$ and the fact that $T_{j} 1=T_{j}^{*} 1=$ $\left(S_{j}-P_{j}\right) 1=\left(S_{j}-P_{j}\right)^{*} 1=0$. For instance, we indicate how to prove that $\left\|T_{j}^{*} Q_{j+k}\right\|_{2-2} \leq C 2^{-\epsilon k}$; for this it suffices to show that $\left\|T_{j}^{*} P_{j+k}\right\|_{2-2} \leq C 2^{-\epsilon k}$. Moreover, by setting $T_{j k}^{*} f(x)=A_{j+k_{*}}^{-1} T_{j}^{*} f(x)$ it is equivalent to the estimate $\left\|T_{j k}^{*} P_{0}\right\|_{2-2} \leq C 2^{-\epsilon k}$. To prove that we just need the cancellation property $T_{j k}^{*} 1=0$ and that $T_{j k}^{*}$ has its distribution kernel supported in $\{(x, y):|x-y| \leq$ $\left.C 2^{-\epsilon k}\right\}$. This reduces to seeing that if $|t| \leq 2^{j+1},\left|x-A_{j+k_{*}}^{-1} \Gamma(x, t)\right| \leq C 2^{-\epsilon k}$. Now

$$
\left|x-A_{j+k_{*}}^{-1} \Gamma(x, t)\right| \leq\left|\frac{t}{2^{j+k}}\right|+\left|\frac{S\left(2^{j+k} x_{1}+t, 2^{j+k} x_{1}\right)}{G\left(2^{j+k}\right)}\right| .
$$

To handle the second term, notice that since $S_{1}^{\prime}(x, x)=0$ then

$$
\begin{aligned}
\left|S\left(2^{j+k} x_{1}+t, 2^{j+k} x_{1}\right)\right|=\mid \int_{0}^{t} S_{1}^{\prime}\left(2^{j+k} x_{1}+s,\right. & \left.2^{j+k} x_{1}\right) d s \mid \\
& \leq \int_{0}^{|t|} A g(E s) d s \leq C G(E|t|),
\end{aligned}
$$

which is smaller than or equal to $C G\left(E 2^{j+1}\right)$. The support condition now follows from the Rivière property. (The estimate $G\left(E 2^{j+1}\right) / G\left(2^{j+k}\right) \leq C 2^{-\epsilon k}$ holds for any $k \geq k_{0}$ with $k_{0}$ such that $2^{k_{0}-1} \geq E$, but otherwise $\left\|T_{j}^{*} Q_{j+k}\right\|_{2-2} \leq C \leq$ $C 2^{-k}$.)

When $k \leq 0$, since $\left\|Q_{j+k} P_{j}^{*}\right\|_{2-2} \leq C 2^{\epsilon k}$ then $\left\|Q_{j+k}\left(S_{j}-P_{j}\right)^{*}\right\|_{2-2} \leq C 2^{\epsilon k}$ is equivalent to $\left\|Q_{j+k} S_{j}^{*}\right\|_{2-2} \leq C 2^{\epsilon k}$, and the bound for $\left\|Q_{j+k} T_{j}^{*}\right\|_{2-2}$ will follow exactly the same argument.

Now we have to break up the operator $S_{j}^{*}$ into two pieces determined by whether or not $t$ is positive, and we work with $\left(\tilde{S}_{j}^{*}\right)=A_{j}^{-1} S_{j}^{*}$. Then we set the normalized "positive" part of the operator $S_{j}^{*}$ as follows

$\left(\tilde{S}_{j}^{*}\right)^{+} f(x)=\int f\left(x_{1}+t, x_{2}+\tilde{S}\left(x_{1}+t, x_{1}\right)\right) \alpha^{+}(t) d t$, with $\tilde{S}(x, y)=\frac{S\left(2^{j} x, 2^{j} y\right)}{G\left(2^{j}\right)}$

where $\alpha^{+}$is a real-valued smoothed-out version of $\chi_{[1,2]}$. (The corresponding kernel for the case $\left(S_{j}^{*}\right)^{-}$is with $\alpha^{-}$being a smoothed-out version of $\left.\chi_{[-2,-1]}\right)$. We write $\tilde{Q}_{j+k}=A_{j}^{-1} Q_{j+k}$. Therefore, we need to show that

$$
\left\|\tilde{Q}_{j+k}\left(\tilde{S}_{j}^{*}\right)^{+}\right\|_{2-2} \leq C 2^{\epsilon k} \text { and } \| \tilde{Q}_{j+k}\left({\tilde{S_{j}^{*}}}^{-} \|_{2-2} \leq C 2^{\epsilon k}\right.
$$


Since the two estimates are similar we concentrate only on the first.

Let $K: \mathbb{R}^{m} \rightarrow C$ be a kernel, and $A: \mathbb{R}^{p} \times \mathbb{R}^{m} \rightarrow \mathbb{R}^{q}$ be a function. Let $T f(x)=\int_{\mathbb{R}^{m}} f\left(x_{1}-y_{1}, x_{2}-A\left(x_{1}, y\right)\right) K(y) d y$ where $\left(x_{1}, x_{2}\right) \in \mathbb{R}^{p} \times \mathbb{R}^{q}$ and $\left(y_{1}, y_{2}\right) \in \mathbb{R}^{p} \times \mathbb{R}^{m-p}$. Define $T_{\lambda} h(x)=\int_{\mathbb{R}^{m}} h\left(x-y_{1}\right) e^{i \lambda A(x, y)} K(y) d y$ where now $x \in \mathbb{R}^{p}$. Then $\left(T S^{*}\right)_{\lambda}=T_{\lambda} S_{\lambda}^{*}$, and Plancherel's theorem in the $x_{2} \in \mathbb{R}^{q}$ variable shows that $\|T\|_{2-2}=\sup _{\lambda}\left\|T_{\lambda}\right\|_{2-2}$. Thus, in our case at hand, it suffices to prove

$$
\left\|\left(\tilde{Q}_{j+k}\right)_{\lambda}\left(\left(S_{j}^{*}\right)^{+}\right)_{\lambda}\right\|_{2-2} \leq C 2^{\epsilon k},
$$

uniformly in $\lambda$, or indeed

$$
\left\|\left(\tilde{Q}_{j+k}\right)_{\lambda}\left(\left(\tilde{S_{j}^{*}}\right)^{+}\right)_{\lambda}\left(\left(\tilde{S_{j}}\right)^{+}\right)_{\lambda}\left(\tilde{Q}_{j+k}^{*}\right)_{\lambda}\right\|_{2-2} \leq C 2^{\epsilon k}
$$

Now the convolution kernel of $\tilde{P}_{j+k}$ can be written as

$$
\frac{1}{2^{k}} \Phi_{1}\left(\frac{x_{1}}{2^{k}}\right) \frac{G\left(2^{j}\right)}{G\left(2^{j+k}\right)} \Phi_{2}\left(\frac{G\left(2^{j}\right) x_{2}}{G\left(2^{j+k}\right)}\right)
$$

for some even functions $\Phi_{1}$ and $\Phi_{2}$ such that $\Phi_{1}$ is supported in $[-2,2]$, and $\Phi_{2}$ is such that $\widehat{\Phi_{2}}$ is identically one in $[-1,1]$ and also supported in $[-2,2]$. By taking the Fourier transform in the second variable we have

$$
\begin{aligned}
\operatorname{Ker}\left(\tilde{Q}_{j+k}\right)_{\lambda}\left(x_{1}\right)=\frac{1}{2^{k}} \Phi_{1}\left(\frac{x_{1}}{2^{k}}\right) \widehat{\Phi_{2}}\left(\frac{G\left(2^{j+k}\right) \lambda}{G\left(2^{j}\right)}\right) & \\
& \quad-\frac{1}{2^{k+1}} \Phi_{1}\left(\frac{x_{1}}{2^{k+1}}\right) \widehat{\Phi_{2}}\left(\frac{G\left(2^{j+k+1}\right) \lambda}{G\left(2^{j}\right)}\right) \\
= & \frac{1}{2^{k+1}} \Phi_{1}\left(\frac{x_{1}}{2^{k+1}}\right)\left[\widehat{C}\left(\frac{G\left(2^{j+k}\right) \lambda}{G\left(2^{j}\right)}\right)-\widehat{\Phi_{2}}\left(\frac{G\left(2^{j+k+1}\right) \lambda}{G\left(2^{j}\right)}\right)\right] \\
& \quad+\frac{1}{2^{k}} \Psi\left(\frac{x_{1}}{2^{k}}\right) \widehat{\Phi_{2}}\left(\frac{G\left(2^{j+k}\right) \lambda}{G\left(2^{j}\right)}\right) \\
\equiv I_{\lambda}\left(x_{1}\right)+I I_{\lambda}\left(x_{1}\right) &
\end{aligned}
$$

where $\Psi(x)=\Phi_{1}(x)-\frac{1}{2} \Phi_{1}\left(\frac{x}{2}\right)$ and so $\int \Psi=0$.

Since $\widehat{\Phi_{2}}$ is identically one in $[-1,1], I_{\lambda}\left(x_{1}\right)=0$ unless $\frac{G\left(2^{j+k+1}\right)|\lambda|}{G\left(2^{j}\right)} \geq 1$, and as $\left|\widehat{\Phi_{2}}\left(\frac{G\left(2^{j+k}\right) \lambda}{G\left(2^{j}\right)}\right)-\widehat{\Phi_{2}}\left(\frac{G\left(2^{j+k+1}\right) \lambda}{G\left(2^{j}\right)}\right)\right| \leq 2$, the estimate (8) when we consider the part $I_{\lambda}$ of the $\operatorname{Ker}\left(\tilde{Q}_{j+k}\right)_{\lambda}$ follows from $\left\|\left(\tilde{Q}_{j+k}^{*}\right)_{\lambda}\right\|_{2-2} \leq C$ and

$$
\left\|\left(\left(S_{j}^{\tilde{*}}\right)^{+}\right)_{\lambda}\left(\left(\tilde{S_{j}}\right)^{+}\right)_{\lambda}\right\|_{2-2} \leq C 2^{\epsilon k}, \quad \text { when } \quad \frac{G\left(2^{j+k+1}\right)|\lambda|}{G\left(2^{j}\right)} \geq 1
$$

It is not difficult to see that the kernel of $\left(\left(\tilde{S}_{j}^{*}\right)^{+}\right)_{\lambda}\left(\left(\tilde{S}_{j}\right)^{+}\right)_{\lambda}$ is, as a function of $x$ and $y$,

$$
K_{\lambda}^{+}(x, y)=\int e^{i \lambda[\tilde{S}(z, y)-\tilde{S}(z, x)]} \alpha^{+}(z-y) \alpha^{+}(z-x) d z
$$


To show (9), since $K_{\lambda}^{+}$is supported in $|x-y| \leq 5$, it suffices to prove that $\int\left|K_{\lambda}^{+}(x, y)\right|^{2} d x \leq C /|\lambda|$ uniformly in $y$, since both the Rivière condition and $G\left(2^{j+k+1}\right)|\lambda| / G\left(2^{j}\right) \geq 1$ then imply $\int\left|K_{\lambda}^{+}(x, y)\right|^{2} d x \leq C 2^{\epsilon k}$. In order to do that we first observe that by Van der Corput's lemma $\left|\bar{K}_{\lambda}(x, y)\right| \leq C /(|\lambda||x-y|)$. Indeed, set $u(z)=\tilde{S}(z, y)-\tilde{S}(z, x)$ then $u^{\prime}(z)=\tilde{S}_{1}^{\prime}(z, y)-\tilde{S}_{1}^{\prime}(z, x)$ and $u^{\prime \prime}(z)=$ $\tilde{S}_{11}^{\prime \prime}(z, y)-\tilde{S}_{11}^{\prime \prime}(z, x)=\tilde{S}_{112}^{\prime \prime \prime}(z, \nu)(y-x)$ for fixed $x$ and $y$ and since $\tilde{S}_{112}^{\prime \prime \prime}$ is singlesigned, $u^{\prime \prime}$ is single-signed and $\left|u^{\prime}(z)\right|=\left|\tilde{S}_{1}^{\prime}(z, y)-\tilde{S}_{1}^{\prime}(z, x)\right| \geq C|x-y|\left|\tilde{S}_{1}^{\prime}(z, x)\right| \geq$ $c|x-y|$ (see Lemma 5 and (6)). Then,

$$
\begin{aligned}
\int\left|K_{\lambda}^{+}(x, y)\right|^{2} d x \leq \int_{\{x:|x-y|<\delta\}} C d x+\int_{\{x:|x-y|>\delta\}} & \frac{1}{\lambda^{2}|x-y|^{2}} d x \\
& \leq C \delta+C \frac{1}{\lambda^{2} \delta} \leq C \frac{1}{|\lambda|}
\end{aligned}
$$

by taking $\delta=1 /|\lambda|$.

Thus the contribution to (8) arising from $I_{\lambda}$ is under control.

Now we need to consider, for technical reasons, separately the cases $\chi_{x \geq y}$ and $\chi_{x \leq y}$. Let $A$ be the operator with kernel $K_{\lambda}^{+}(x, y) \chi_{\{x \geq y\}}$ and let $B$ be the operator with kernel $K_{\lambda}^{+}(x, y) \chi_{\{x \leq y\}}$; since $\overline{K_{\lambda}^{+}}(y, x)=K_{\lambda}^{+}(x, y)$ we have that $A^{*}=B$, and in order to prove (8) it is enough to prove it either for $A$ or for $B$. Since we have a trivial estimate for the $L^{\infty}$ operator norm it suffices to show that the $L^{1}$ norm of the operator has the decay we want, and in fact it is enough to show that

$$
\int\left|\int I I_{\lambda}\left(x-x^{\prime}\right) K_{\lambda}^{\prime}\left(x^{\prime}, y\right) d x^{\prime}\right| d x \leq C 2^{\epsilon k}
$$

uniformly in $y \in \mathbb{R}, \lambda \in \mathbb{R}$, and $K_{\lambda}^{\prime}$ denotes $K_{\lambda}^{+}$restricted to $x \geq y$. But $\int I I_{\lambda}\left(x-x^{\prime}\right) K_{\lambda}^{\prime}\left(x^{\prime}, y\right) d x^{\prime}=C \Psi_{k} *_{1} K_{\lambda}^{\prime}(\cdot, y)$ (*1 means convolution in the first variable and $\left.\Psi_{k}(x)=\frac{1}{2^{k}} \Psi\left(\frac{x}{2^{k}}\right)\right)$, and therefore since $\int \Psi_{k}=0$ the following lemma finishes the proof.

Lemma 6. For $K_{\lambda}^{\prime}(x, y)=K_{\lambda}^{+}(x, y) \chi_{\{x \geq y\}}(y)$, we have

$$
\int\left|K_{\lambda}^{\prime}(x+h, y)-K_{\lambda}^{\prime}(x, y)\right| d x \leq C|h|^{\frac{1}{2}}
$$

Proof. Let us assume $|h| \leq \frac{1}{4}$ otherwise the conclusion is clear, and let us assume 
for simplicity that $h>0$. Then

$$
\begin{aligned}
& \int\left|K_{\lambda}^{\prime}(x+h, y)-K_{\lambda}^{\prime}(x, y)\right| d x \\
& \leq \int\left|\int\left[e^{i \lambda[\tilde{S}(z, y)-\tilde{S}(z, x+h)]}-e^{i \lambda[\tilde{S}(z, y)-\tilde{S}(z, x)]}\right] \alpha^{+}(z-y) \alpha^{+}(z-x-h) d z\right| d x \\
& \quad+\int \mid \int e^{i \lambda[\tilde{S}(z, y)-\tilde{S}(z, x)]}\left(\alpha^{+}(z-y) \alpha^{+}(z-x)\right. \\
& =I+I I .
\end{aligned}
$$

The second term is fine because, since we are working with normalized pieces of curves the regions of integration are finite, and the function $\alpha^{+}$is smooth enough, so it is clearly $O(|h|)$. The first term satisfies

$$
\begin{aligned}
I & =\int\left|\iint_{0}^{h} \frac{\partial}{\partial t} e^{i \lambda[\tilde{S}(z, y)-\tilde{S}(z, x+t)]} d t \alpha^{+}(z-y) \alpha^{+}(z-x-h) d z\right| d x \\
& =\int\left|\iint_{0}^{h} i \lambda \tilde{S}_{2}^{\prime}(z, x+t) e^{i \lambda[\tilde{S}(z, y)-\tilde{S}(z, x+t)]} d t \alpha^{+}(z-y) \alpha^{+}(z-x-h) d z\right| d x \\
& \leq|h| \sup _{0 \leq t \leq h} \int\left|\int \lambda \tilde{S}_{2}^{\prime}(z, x+t) e^{i \lambda[\tilde{S}(z, y)-\tilde{S}(z, x+t)]} \alpha^{+}(z-y) \alpha^{+}(z-x-h) d z\right| d x \\
& =|h| \sup _{0 \leq t \leq h} \int\left|\int \lambda \tilde{S}_{2}^{\prime}(z, x) e^{i \lambda[\tilde{S}(z, y)-\tilde{S}(z, x)]} \alpha^{+}(z-y) \alpha^{+}(z-x-h+t) d z\right| d x .
\end{aligned}
$$

Then, it suffices to show that

$$
\int_{x:|x-y|>\delta}\left|\int \lambda \tilde{S}_{2}^{\prime}(z, x) e^{i \lambda[\tilde{S}(z, y)-\tilde{S}(z, x)]} \alpha^{+}(z-y) \alpha^{+}(z-x-h+t) d z\right| d x \leq \frac{C}{\delta},
$$

independently of $0 \leq t \leq h$, because then

$$
\int\left|K_{\lambda}^{\prime}(x+h, y)-K_{\lambda}^{\prime}(x, y)\right| d x \leq C \delta+C \frac{|h|}{\delta} \leq C|h|^{\frac{1}{2}}
$$

by taking $\delta=|h|^{\frac{1}{2}}$. Now we integrate by parts with respect to $z$ and obtain

$$
\begin{aligned}
\int \lambda & \lambda \tilde{S}_{2}^{\prime}(z, x) e^{i \lambda[\tilde{S}(z, y)-\tilde{S}(z, x)]} \alpha^{+}(z-y) \alpha^{+}(z-x-h+t) d z \\
= & -\frac{1}{i} \int \frac{\partial}{\partial z}\left(\frac{\tilde{S}_{2}^{\prime}(z, x)}{\tilde{S}_{1}^{\prime}(z, y)-\tilde{S}_{1}^{\prime}(z, x)}\right) e^{i \lambda[\tilde{S}(z, y)-\tilde{S}(z, x)]} \alpha^{+}(z-y) \alpha^{+}(z-x-h+t) d z \\
& -\frac{1}{i} \int \frac{\partial}{\partial z}\left(\alpha^{+}(z-y) \alpha^{+}(z-x-h+t)\right) \frac{\tilde{S}_{2}^{\prime}(z, x)}{\tilde{S}_{1}^{\prime}(z, y)-\tilde{S}_{1}^{\prime \prime}(z, x)} e^{i \lambda[\tilde{S}(z, y)-\tilde{S}(z, x)]} d z .
\end{aligned}
$$


Since for $K_{\lambda}^{\prime}(x, y)=K_{\lambda}^{+}(x, y) \chi_{\{x \geq y\}}(y)$ we are under the hypothesis of Lemma 5 and then we get $\left|\tilde{S}_{1}^{\prime}(z, y)-\tilde{S}_{1}^{\prime}(z, x)\right| \geq C|x-y|\left|\tilde{S}_{2}^{\prime}(z, x)\right|$. We shall be finished if we can show

$$
\int_{x:|x-y|>\delta} \int\left|\frac{\partial}{\partial z}\left(\frac{\tilde{S}_{2}^{\prime}(z, x)}{\tilde{S}_{1}^{\prime}(z, y)-\tilde{S}_{1}^{\prime}(z, x)}\right)\right| d z d x \leq \frac{C}{\delta}
$$

for $1<z-y<2$ and $0<z-x<3$ (recall that $0<t<h$ and $h \leq \frac{1}{4}$ ). But

$$
\begin{aligned}
\frac{\partial}{\partial z} \frac{\tilde{S}_{2}^{\prime}(z, x)}{\tilde{S}_{1}^{\prime}(z, y)-\tilde{S}_{1}^{\prime}(z, x)} & =\frac{-\tilde{S}_{12}^{\prime \prime}(z, x) \tilde{S}_{1}^{\prime}(z, x)}{\left[\tilde{S}_{1}^{\prime}(z, y)-\tilde{S}_{1}^{\prime}(z, x)\right]^{2}}+\frac{\tilde{S}_{12}^{\prime \prime}(z, x) \tilde{S}_{1}^{\prime}(z, y)}{\left[\tilde{S}_{1}^{\prime}(z, y)-\tilde{S}_{1}^{\prime}(z, x)\right]^{2}} \\
& -\frac{\tilde{S}_{2}^{\prime}(z, x)\left[\tilde{S}_{11}^{\prime \prime}(z, y)-\tilde{S}_{11}^{\prime \prime}(z, x)\right]}{\left[\tilde{S}_{1}^{\prime}(z, y)-\tilde{S}_{1}^{\prime}(z, x)\right]^{2}} \\
& =M+N+L .
\end{aligned}
$$

Now, it is very important to check that each of the terms has single sign and that $\operatorname{sgn} M=\operatorname{sgn} L$; fortunately we know the signs precisely in terms of the sign of $\tilde{S}_{112}^{\prime \prime \prime}\left(\right.$ we use that $\tilde{S}_{11}^{\prime \prime}(z, y)-\tilde{S}_{11}^{\prime \prime}(z, x)=\tilde{S}_{112}^{\prime \prime \prime}(z, \nu)(y-x)$.) Indeed then, by (4) and (5)

$$
\begin{aligned}
\operatorname{sgn} M & =\operatorname{sgn}\left(-\tilde{S}_{12}^{\prime \prime}(z, x) \tilde{S}_{1}^{\prime}(z, x)\right)=\operatorname{sgn} \tilde{S}_{112}^{\prime \prime \prime} \operatorname{sgn}(x-z)\left(-\operatorname{sgn} \tilde{S}_{112}^{\prime \prime \prime}\right) \\
& =\operatorname{sgn}(z-x), \\
\operatorname{sgn} N & =\operatorname{sgn}\left(\tilde{S}_{12}^{\prime \prime}(z, x) \tilde{S}_{1}^{\prime}(z, y)\right)=-\operatorname{sgn} \tilde{S}_{112}^{\prime \prime \prime} \operatorname{sgn}(x-z)\left(-\operatorname{sgn} \tilde{S}_{112}^{\prime \prime \prime}\right) \\
& =\operatorname{sgn}(x-z), \quad \text { and } \\
\operatorname{sgn} L & =-\operatorname{sgn} \tilde{S}_{112}^{\prime \prime \prime} \operatorname{sgn} \tilde{S}_{112}^{\prime \prime \prime} \operatorname{sgn}(y-x) \\
& =\operatorname{sgn}(x-y) .
\end{aligned}
$$

So since $z-x>0$ then $M$ and $N$ have single sign. Also since we need to prove the lemma only for $K_{\lambda}^{\prime}(x, y)=K_{\lambda}^{+}(x, y) \chi_{\{x \geq y\}}(y)$ then $\operatorname{sgn} M=\operatorname{sgn} L$. Therefore, we can use that

$$
\iint|M+N+L| d z d x \leq\left|\iint M d z d x+\iint L d z d x\right|+\left|\iint N d z d x\right| .
$$

The double integral of $N$ is, for some boundary points $x^{*}$ and $x^{* *}$, by using Lemma 5 and the fact that we are always integrating over bounded intervals, controlled by

$$
\left.\left|\iint N d x d z\right| \leq \int \mid \frac{\tilde{S}_{1}^{\prime}(z, y)}{\tilde{S}_{1}^{\prime}(z, y)-\tilde{S}_{1}^{\prime}(z, x)}\right]_{x^{*}}^{x^{* *}} \mid d z \leq \frac{C}{\delta} \int d z \leq \frac{C}{\delta} .
$$


Now we integrate $M$, first in the variable $x$ and then with respect to $z$,

$$
\left.\iint M d x d z=\int \frac{-\tilde{S}_{1}^{\prime}(z, x)}{\tilde{S}_{1}^{\prime}(z, y)-\tilde{S}_{1}^{\prime}(z, x)}\right]_{x^{*}}^{x^{* *}} d z+\iint \frac{\tilde{S}_{12}^{\prime \prime}(z, x)}{\tilde{S}_{1}^{\prime}(z, y)-\tilde{S}_{1}^{\prime}(z, x)} d x d z .
$$

In the same way, but first in the variable $z$ and then with respect to $x$,

$$
\left.\iint L d z d x=\int \frac{\tilde{S}_{2}^{\prime}(z, x)}{\tilde{S}_{1}^{\prime}(z, y)-\tilde{S}_{1}^{\prime}(z, x)}\right]_{z^{*}}^{z^{* *}} d x-\iint \frac{\tilde{S}_{12}^{\prime \prime}(z, x)}{\tilde{S}_{1}^{\prime}(z, y)-\tilde{S}_{1}^{\prime}(z, x)} d z d x,
$$

for suitable boundary points $z^{*}$ and $z^{* *}$. Therefore, again Lemma 5 gives

$$
\left.\left.\left|\iint(M+L) d z d x\right|=\mid \int \frac{-\tilde{S}_{1}^{\prime}(z, x)}{\tilde{S}_{1}^{\prime}(z, y)-\tilde{S}_{1}^{\prime}(z, x)}\right]_{x^{*}}^{x^{* *}} d z+\int \frac{\tilde{S}_{2}^{\prime}(z, x)}{\tilde{S}_{1}^{\prime}(z, y)-\tilde{S}_{1}^{\prime}(z, x)}\right]_{z^{*}}^{z^{* *}} d x \mid \leq \frac{C}{\delta},
$$

as required.

\section{References}

[C] A. Carbery, A version of Cotlar's lemma for $L^{p}$ spaces and some applications, Contemp. Math. 189 (1995), 117-134.

[CCVWW] A. Carbery, M. Christ, J. Vance, S. Wainger and D. Watson, Operators associated to flat plane curves: $L^{p}$ estimates via dilation methods, Duke Math. J. 59 (1989), 675-700.

[CCW] A. Carbery, M. Christ and J. Wright, Multidimensional van der Corput and sublevel set estimates, J. Amer. Math. Soc., to appear.

[CVWW] A. Carbery, J. Vance, S. Wainger and J. Wright, A variant of the notion of a space of homogeneous type, J. Funct. Anal. 132 (1995), 119-140.

[CWW1] A. Carbery, S. Wainger and J. Wright, Hilbert transforms and maximal functions associated to flat curves, J. Fourier Anal. Appl. Special Issue (1995), 119-139.

[CWW2] A. Carbery, S. Wainger and J. Wright, Hilbert transforms and maximal functions associated to flat curves on the Heisenberg group, J. Amer. Math. Soc. $\mathbf{8 , 1}$ (1995), 141-179.

[CCCDRVWW] H. Carlsson, M. Christ, A. Córdoba, J. Duoandikoetxea, J.L. Rubio de Francia, J. Vance, S. Wainger and D. Weinberg, $L^{p}$ estimates for maximal functions and Hilbert transforms along variable flat convex curves in $\mathbb{R}^{2}$, Bull. Amer. Math. Soc. (N.S.) 14 (1986), 263-267.

[CNSW] M. Christ, A. Nagel, E. M. Stein and S. Wainger, Singular and maximal Radon transforms: analysis and geometry., preprint, to appear.

[DR] J. Duoandikoetxea and J.L. Rubio de Francia, Maximal and singular integral operators via Fourier transform estimates, Invent. Math. 84 (1986), 541-561.

[NVWW1] A. Nagel, J. Vance, S. Wainger and D. Weinberg, Hilbert transforms for convex curves, Duke Math. J. 50 (1983), 735-744.

[NVWW2] A. Nagel, J. Vance, S. Wainger and D. Weinberg, Maximal functions for convex curves, Duke Math. J. 52 (1985), 715-722.

[P] Y. Pan, Uniform estimates for oscillatory integral operators, J. Funct. Anal. 100 (1991), 207-220. 
[PS] D.H. Phong and E.M. Stein, Hilbert integrals, singular integrals and Radon transforms,I, Acta Math. 157 (1986), 99-157.

[PS1] D.H. Phong and E.M. Stein, On a stopping process for oscillatory integrals, J. Geom. Anal. 4 (1994), 104-120.

[S] A. Seeger, $L^{2}$ estimates for a class of singular oscillatory integrals, Math. Res. Lett. 1 (1994), 65-73.

Department of Mathematics and Statistics, University of Edinburgh, James Clerk Maxwell Building, The King's Buildings, Mayfield Rd., Edinburgh EH9 3JZ, U.K.

E-mail address: carbery@maths.ed.ac.uk

Departamento de Matemáticas, Facultad de Ciencias, Universidad Autónoma de MADRID, 28049 MADRID, SPAIN

E-mail address: sonsoles.perez@uam.es 